\title{
Outcomes of Total Parathyroidectomy with Autotransplantation versus Subtotal Parathyroidectomy with Routine Addition of Thymectomy to both Groups: Single Center Experience of Secondary Hyperparathyroidism
}

\author{
Gürhan Sakman ${ }^{1}$, Cem Kaan Parsak¹, Mustafa Balal ${ }^{2}$, Gülşah Seydaoglu ${ }^{3}$, İsmail Cem Eray ${ }^{1}$, \\ Gökhan Sarıtaş ${ }^{1}$, Orhan Demircan ${ }^{4}$
}

\begin{abstract}
${ }^{1}$ Department of General Surgery, Çukuruva University Faculty of Medicine, Adana, Turkey
${ }^{2}$ Department of Nephrology, Çukuruva University Faculty of Medicine, Adana, Turkey

${ }^{3}$ Department of Biostatistics, Çukuruva University Faculty of Medicine, Adana, Turkey ${ }^{4}$ Department of General Surgery, Acıbadem Hospital, Adana, Turkey
\end{abstract}

\begin{abstract}
Background: Secondary hyperparathyroidism is a common acquired disorder seen in chronic renal failure. It may result in potentially serious complications including metabolic bone diseases, severe atherosclerosis and undesirable cardiovascular events. Parathyroidectomy is required in about $20 \%$ of patients after 3-10 years of dialysis and in up to $40 \%$ after 20 years.

Aims: The aim of the current study was to evaluate the short-term and long-term outcomes of patients with secondary hyperparathyroidism who had undergone total parathyroidectomy with autotransplantation and thymectomy or subtotal parathyroidectomy with thymectomy by the same surgical team during the study period.

Study Design: Retrospective comparative study.

Methods: Clinical data of 50 patients who underwent parathyroid surgery for secondary hyperparathyroidism between 2003 and 2011 were reviewed retrospectively. Patients were divided into two subgroups of total parathyroidectomy with autotransplantation or subtotal parathyroidectomy. Thymectomy was routinely performed for both groups. Short term outcome parameters included intact parathyroid hormone, ionized calcium and alkaline phosphatase levels. Bone pain, bone fractures, persistent or recurrent disease were included in long term outcome parameters.
\end{abstract}

Results: The mean duration of dialysis was eight years. The mean ionized calcium levels dropped significantly in the total parathyroid- ectomy with autotransplantation group $(\mathrm{p}=0.016)$. No serious postoperative complications were observed. Postoperative intravenous calcium supplementation was required in four patients in the total parathyroidectomy with autotransplantation group (total PTX+AT) and in three patients in the subtotal parathyroidectomy group (subtotal PTX). Postoperatively, all patients received oral calcium carbonate and calcitriol. The length of average hospital stay was 5 (3-10) days. Including nine patients who underwent successful renal transplantation pre-operative bone symptoms, hypercalcemia, hyperphosphatemia, and an increased alkaline phosphatase levels were improved or resolved in all patients. After a mean follow-up of 65 months, three patients $(6 \%)$ had persistent and one $(2 \%)$ had recurrent disease.

Conclusion: Total parathroidectomy with autotransplantation is a beneficial and safe surgical procedure for patients on chronic dialysis with otherwise uncontrollable secondary hyperparathroidism and even in patients who have undergone renal transplantation after parathyroidectomy. Careful cervical exploration and routine thymectomy should be considered as a routine part of the surgical approach regardless of the preferred technique.

(Balkan Med J 2014;31:77-82).

Key Words: Autotransplantation, chronic renal failure, secondary hyperparathyroidism, parathyroidectomy, thymectomy
Secondary hyperparathyroidism (sHPT) is a common acquired disorder seen in chronic renal failure. It may result in potentially serious complications including metabolic bone diseases, severe atherosclerosis and undesirable cardiovascular events (1). Parathyroidectomy (PTX) is required in about $20 \%$ of patients after 3-10 years of dialysis and in up to $40 \%$ after 20 years $(2,3)$
When medical treatment fails, subtotal parathyroidectomy (subtotal PTX) with a cervical remnant and total parathyroidectomy with autotransplantation (total PTX+AT) are the standard procedures, although both are associated with high recurrence rates (4-7). A considerable quantity of functioning parathyroid tissue is left after each of these operations in order to prevent possible hypoparathyroidism and the need for long- 
term calcium and vitamin D supplementation (4); it is this practice that may result in recurrence because of growth of the parathyroid remnant or the autograft (6). The third surgical option is total parathyroidectomy without an autoimplant, which has been described and reported to be potentially associated with complications such as adynamic bone disease and hypocalcaemia $(4,8)$. Recently, this technique has gained popularity again, but there is still controversy about its outcomes, and long-term studies are required.

The aim of the current study was to evaluate the short-term and long-term outcomes of patients with secondary hyperparathyroidism who had undergone total parathyroidectomy with autotransplantation and thymectomy or subtotal parathyroidectomy with thymectomy by the same surgical team during the study period.

\section{MATERIAL AND METHODS}

This study was approved by the Institutional Review Board of Çukurova University Faculty of Medicine, Adana, Turkey. All patients enrolled in the study were informed about the aim of the study and provided written informed consent.

\section{Study population}

This prospective study recruited patients who were treated at the Nephrology and General Surgery Clinics between June 2003 and December 2011 and had undergone total PTX with AT or subtotal PTX due to SHPT because of renal disease.

The diagnosis of severe SHPT was established in all of the patients on the basis of clinical, biochemical, radiological and histological evidence. Our inclusion criteria for parathyroidectomy in symptomatic patients with sHPT included: age equal to or greater than 18 years, long-term dialysis treatment ( $>12$ months), very high levels of intact parathyroid hormone (iPTH) $(>500 \mathrm{pg} / \mathrm{mL})$ that could not be normalized with the drug treatment available at the time, enlarged parathyroid glands visualized through imaging techniques, findings indicating osteitis fibrosa cystica or high bone turnover and at least one factor refractory to medical treatment (hypocalcaemia, serum calcium-phosphate production over $70 \mathrm{mg} / \mathrm{dl}$, osteodystrophy or calciphylaxis, bone and joint pain or pathological fracture). Patients with a history of neck explorations for thyroid/parathyroid disorders or malignant disease of the thyroid glands were excluded from the study.

\section{Study design and data collection}

Patients were divided into surgical groups of total PTX+AT and subtotal PTX patients. Preoperative localization studies included neck ultrasonography and Tc-99 m sestamibi parathyroid scans. Serumionized calcium (iCa), phosphorus (P), alkaline phosphatase (AP) and intact parathyroid hormone (iPTH) were measured before parathyroidectomy and yearly after surgery in all patients from both groups.

\section{Surgical approach}

In all patients, the neck and presternal area were explored without sternotomy for the identification of the four parathyroid glands. Tran- scervical thymectomy and bilateral exploration of the carotid sheath were routinely performed. In cases of subtotal parathyroidectomy, a small cervical remnant of the most normal-appearing inferior gland was left in situ and signed with a small clip. In the total PTX group, the most normal-appearing non-nodular areas of the parathyroid gland were divided into pieces $1 \mathrm{~mm}^{3}$ in size, and 15-20 of these pieces were placed in a single muscle pocket of the brachioradialis muscle and signed with a clip. As a result, for total parathyroidectomy with autotransplant, 50-60 mg of parathyroid tissue was autografted to the forearm; for subtotal parathyroidectomy, approximately 40-50 mg of parathyroid tissue was left in situ. All parathyroid tissues that were resected, autotransplanted, or left in situ were confirmed histologically. Intraoperative PTH measurements (IOPTH) in order to confirm surgical cure were available in only 23 patients. Our local population is particularly susceptible to thyroid disorders; therefore, thyroidectomy was performed whenever there was accompanying nodular thyroid disease. All surgical procedures were performed at the same center by the same surgical team throughout the study period.

During the first 48 postoperative hours, postoperative hypocalcaemia was managed with intravenous injections of $10 \%$ calcium gluconate given in response to actual serum calcium concentrations or severe symptoms or signs of hypocalcaemia. Patients were discharged from the hospital as soon as serum calcium levels were stabilized with oral calcium and cholecalciferol therapy. During the subsequent follow-up period, calcium and calcitriol supplements were adjusted orally according to each patient's serum calcium concentration.

\section{Follow-up}

Each patient was re-evaluated in a clinical follow-up visit at a general surgery outpatient clinic or, alternatively, in a telephone interview while his or her laboratory data for follow-up were provided by the nephrologist in charge of his or her case. Bone mineral density was measured for all patients using dual energy X-ray absorptiometry before parathyroidectomy and yearly afterward. All patients were monitored for fractures of any kind.

We define hypoparathyroidism as an iPTH measurement less than $12 \mathrm{pg} / \mathrm{mL}$ persisting one year after parathyroidectomy with normal or low serum calcium levels under vitamin $\mathrm{D}$ and oral calcium replacement. Hyperparathyroidism recurrence after surgery is defined as a high level of intact PTH persisting throughout the late postoperative follow-up period that fails to respond to medical/pharmacological management. We used the National Kidney Foundation's Kidney Disease Outcomes Quality Initiative (K-DOQI) guidelines to define persistent or recurrent high iPTH (300 pg/mL) (9).

\section{Statistical analysis}

For each continuous variable, normality was checked by histograms and Shapiro Wilk or Kolmogorov-Smirnov normality tests. Parametric tests were chosen for the continuous data that were normally distributed. Since the data were not distributed normally, an appropriate non-parametric test was chosen. Comparisons between groups were performed using Student's t-test for normally distributed data and the Mann-Whitney $U$ test for data that were not normally distributed. Time-dependent intragroup data were analyzed by the Friedman test. The categorical variables between the groups were analyzed using the Chi square test or Fisher's exact test. Results were presented as mean $\pm \mathrm{SD}$ and median (min-max). Statistical analyses were performed using the statistical package SPSS v 19.0. 


\section{RESULTS}

This study randomly divided 50 patients, 25 female and 25 male, into two groups: a total PTX+AT group with a mean age \pm SD of $36.4 \pm 11.7$ years (range: $18-63$ ) and a subtotal PTX group with a mean age \pm SD of $42.6 \pm 14.5$ (range: $18-71$ ) years. The demographic and clinical data of these patient groups are summarized in Table 1. There were no statistically significant differences between the groups. The average follow-up period was 65 months (range: 13-118).

In addition to parathyroidectomy, thyroidectomy was performed on nine patients (total thyroidectomy $=6$ and lobectomy + isthmectomy $=3$ ) because of coexisting thyroid disease. The majority of the removed parathyroid glands were found in normal anatomical positions, although some glands were found within the thymus $(n=12)$, in the carotid sheath $(n=4)$ and in the paraesophageal region $(n=1)$. Two total thyroidectomies and one lobectomy + isthmectomy were performed in the total PTX+AT group while four total thyroidectomies and two lobectomy + isthmectomy operations were performed in the subtotal PTX group. There were no perioperative complications in either group. The average length of hospital stay was 5 (range: 3-10) days. Postoperatively, no vocal cord paralysis or substantial bleeding, hematoma or seroma requiring reoperation was recorded.

The only significant difference between the groups was in serum ionized calcium levels 24 hours postoperation $(p=0.016)$. As expected, postoperative calcium levels were

TABLE 1. Characteristics of patients and laboratory findings

\begin{tabular}{lccc}
\hline & \multicolumn{3}{c}{ Groups } \\
& $\begin{array}{c}\text { Total PTX+AT } \\
(\mathrm{n}=25)\end{array}$ & $\begin{array}{c}\text { Subtotal PTX } \\
(\mathrm{n}=25)\end{array}$ & $\mathrm{p}$ \\
\hline Sex (Male/Female) & $10 / 15$ & $15 / 10$ & 0.258 \\
Age, Years* & $36.4 \pm 11.7$ & $42.6 \pm 14.5$ & 0.107 \\
& $36(18-63)$ & $43(18-71)$ & \\
Years on Dialysis* & $8.4 \pm 5.3$ & $9.9 \pm 5.5$ & 0.258 \\
& $7(2-20)$ & $9(3-21)$ & \\
Preoperative Phosphorus* & $5.7 \pm 1.2$ & $5.6 \pm 1.2$ & 0.331 \\
& $5.5(3.3-8.6)$ & $5.2(3.7-8.2)$ & \\
Preoperative Alkaline & $1127 \pm 615$ & $1074 \pm 872$ & 0.295 \\
Phosphatase* & $987(367-2940)$ & $859(362-4428)$ \\
Preoperative Serum-Ionized & $1.44 \pm 0.1$ & $1.46 \pm 0.1$ & 0.382 \\
Calcium* & $1.40(1.32-1.68)$ & $1.42(1.34-1.76)$ \\
Postoperative Serum-Ionized & $0.83 \pm 0.12$ & $0.91 \pm 0.12$ & 0.016 \\
Calcium * & $0.84(0.56-1.00)$ & $0.95(0.62-1.10)$ \\
Follow-Up Duration (Months)* & $66.84 \pm 32.5$ & $64.44 \pm 33.0$ & 0.786 \\
& $67(14-118)$ & $60(13-117)$ & \\
Presence of Thyroid Nodules, $\mathrm{n}(\%)$ & $3(12.5)$ & $6(24.0)$ & 0.463 \\
Number of Transplantations, $\mathrm{n}(\%)$ & $5(20.0)$ & $4(16.0)$ & 1.000 \\
Alive/Dead (\%) & $22 / 3(12.0)$ & $23 / 2(8.0)$ & 1.000 \\
\hline
\end{tabular}

Total PTX+AT : total parathyroidectomy with autotransplantation; subtotal PTX: subtotal parathyroidectomy

*Data expressed as mean $\pm \mathrm{SD}$ and median (min-max)

Reference values: Serum-ionised calcium: 1.11-1.40 mmol/L; phosphorus: 2.3-4.6 mg/dL; alkaline phosphatase: $35-129 \mathrm{U} / \mathrm{L}$. lower in the total PTX group, but they eventually increased to within the reference range. Postoperative intravenous calcium supplementation (up to a maximum of $0.8 \mathrm{~g} / \mathrm{kg} / \mathrm{day}$ ) was required in four patients from the total PTX+AT group and three patients from the subtotal PTX group. Postoperatively, all patients were supplemented with active vitamin D analogues and temporarily with cholecalciferol $1 \mathrm{~g} /$ day (for 4-6 weeks). Active vitamin $\mathrm{D}$ was then given in a median daily dose of 0.5 g calcitriol (range: 0-2.5 g/day). There was no serious clinical problems related to hypocalcaemia, even in patients with symptomatic hypocalcaemia.

The serum iPTH levels decreased progressively during the first postoperative week, and the clinical symptoms including bone pain were improved in all formerly symptomatic patients. The individual courses of iPTH changes in the two groups after surgery are shown in Figure 1. The short-term correction of severe hyperparathyroidism was similar in the two surgical patient groups, reaching $100 \%$ in the total PTX+AT group and $92 \%$ in the subtotal PTX group. One patient six years removed from his total PTX+AT and two patients nine years removed from subtotal PTX operations were the only patients to exhibit serum iPTH levels under normal reference values. During our follow-up period, none of the patients had iPTH levels above $500 \mathrm{pg} / \mathrm{mL}$. One patient in the total PTX+AT group had hyperparathyroidism (not above $130 \mathrm{pg} / \mathrm{mL}$ ) after five years of follow-up, and two patients in the subtotal PTX group had persistent parathyroid over function (one with $98 \mathrm{pg} / \mathrm{mL}$ at eight years after surgery, one with $108 \mathrm{pg} / \mathrm{mL}$ at nine years). One case experienced a recurrence of hyperparathyroidism that responded to medical therapy consisting of calcimimetics without any requirement for surgery.

The occurrence of hypoparathyroidism was observed in patients in both groups with total thyroidectomy. Although the sample size was insufficient to allow us to draw firm conclusions, the mean level of iPTH was found to be lower in the subtotal PTX group with thyroidectomy than in the total PTX+AT group with thyroidectomy or than the other groups (Figure 2).

Renal transplantation was performed successfully in nine patients, four of whom were from the subtotal PTX group. When we compared the patients who underwent renal transplantation with other patients, there was no statistically significant difference between the groups in terms of iPTH levels (Figure 3).

\section{DISCUSSION}

This retrospective study showed that recurrence rates in patients with SHPT were lower after total parathyroidectomy with autotransplantation than after subtotal parathyroidectomy. The effectiveness of routine thymectomy was also demonstrated. Finally, the findings of the present study show that total PTX+AT or subtotal PTX results in a dramatic reduction 
in the serum iPTH level, an improvement in sHPT, and a decrease in complication rates and bone pain.

The rates of symptomatic hyperparathyroidism and parathyroidectomy increase with time on dialysis treatment and with survival time (10). In our country, we still have difficulties in providing kidney transplantation for all patients with chronic renal failure, so that many are exposed to the undesirable effects of long-term dialysis treatment. The prevalence of parathyroidectomy in end stage renal disease ranges from $9.2 \%$ after $10-15$ years of dialysis to $20.8 \%$ after $16-20$ years of dialysis $(11,12)$.

Vitamin D analogues and calcimimetics have demonstrated efficacy, but this prophylactic therapy is not always effective and is also expensive. In some patients, cinacalcet may delay the need for PTX until the patient gets a renal transplant. In all others, active vitamin D, cinacalcet and PTX can be used sequentially. However, cinacalcet is not a cost-effective treatment for patients with sHPT because of its high cost $(13,14)$. Unfortunately, during our study period (2003-2011), no calcimimetics were available in our country. Advances in pharmacotherapy and dialysis continue, but the medical management of this condition remains suboptimal and expensive, especially in Turkey.

The success of surgical techniques for secondary hyperparathyroidism is correlated with success in the identification and removal of all parathyroid glands. In the preoperative course, identifying the localization of hyperplasic glands with ${ }^{99 \mathrm{~m}} \mathrm{Tec}$ sestamibi and ultrasonography may be insufficient (12-15). The presence of aberrant localization or an ectopic gland observed through scintigraphy may affect the success of the exploration (16). Milas et al. (17) reported that approximately $38 \%$ of mediastinal ectopic glands may be observed through scintigraphy. In most cases, identifying the localization of all glands using only these two routine imaging techniques in the preoperative course may be impossible (18). The reason for this is the decreased sensitivity of imaging techniques in multiglandular parathyroid diseases apart from etiology. In our study, we detected neither aberrant glands nor any cases in which all four glands were preoperatively identified. Neither of the techniques were found to be superior to the other, but the simultaneous use of both techniques increased the number of glands that could be identified. Nevertheless, preoperative ultrasonography helped in the detection of co-existent thyroid nodules in addition to revealing the localization of the parathyroid glands.

To avoid persistent/recurrent hyperparathyroidism in surgery for sHPT, thymectomy should be performed routinely during the first surgical exploration from the cervical incision because basically all parathyroid glands are hyperplasic in these patients. The effectiveness of imaging is important for the surgeon only when an ectopic gland is identified. The success of sHPT surgery depends on the effective removal of all hyperplasic parathyroid glands and the pathological confirmation that all parathyroid tissues that were resected, autotrans- planted or retained in the neck $(1,2,5)$. We routinely perform cervical thymectomies and freeze and section all removed glands and tissues for perioperative confirmation. Through pathological evaluations, we also identify glands with diffuse hyperplasia, which we prefer for autotransplantation or to be left in the neck. It has been reported that patients with parathyroid nodular hyperplasia have higher rates of recurrence than those with diffuse hyperplasia after subtotal PTX or total PTX+AT (15). The cost of frozen section analysis is almost equal to that of IOPTH testing at our hospital. We do not routinely study IOPTH levels for patients with SHPT, because the benefit of IOPTH data in SHPT has not been clearly defined. IOPTH measurement by means of rapid intact PTH assays is not recommended for routine use in SHPT patients, as the intraoperative characteristics of intact PTH levels cannot predict early or long-term outcomes (16-18).

In the literature, the preferred autotransplantation locations after total parathyroidectomy are identified as the anterior brachioradial muscle, the sternocleidomastoid (SCM) muscle and the presternal area, where the pectoralis major muscle enters the sternum at the level of the left second intercostal space (10). The arm chosen for the transplantation into the brachioradial muscle should preferably have no pre-existing or planned arteriovenous fistula. Transplantation is generally performed on the dominant arm in order to reserve the non-dominant arm for the fistula, offering the advantageous possibility of a minimally invasive surgical intervention with local anesthesia in case of recurrence due to autograft. We prefer the arm as a site for autotransplantation because the alternative approach in the neck may be more difficult, especially with local anesthesia. It is also more difficult to determine the localization in cases of transplantation to the neck (SCM muscle) or presternal muscle and to differentiate any supernumeric parathyroid glands in the neck or the mediastinum by means of MIBI in the event of any recurrence due to the graft. In the anterior arm, on the other hand, MIBI activity is usually diagnostic.

At the routine one-month postoperative iPTH level examination in the parathyroid autotransplant group, all patients presented normalization of iPTH levels except for one patient with an iPTH level lower than $12 \mathrm{pg} / \mathrm{ml}$, whose hypoparathyroidism was observed to persist at subsequent examinations as well. This indicates that normalization should be expected within the first four weeks after the operation, although our data do not permit us to claim this with certainty. In the literature, the mean normalization time of PTH is reported as two weeks after the operation $(19,20)$.

As there is no consensus concerning what constitutes adequate iPTH levels in patients with chronic renal failure, the prevalence of late hypoparathyroidism has been defined in the literature as ranging from $3 \%$ to $38 \%(21,22)$. In our study, late hypoparathyroidism after the operation occurred in three (6\%) of 50 patients. The large range given for prevalence may 


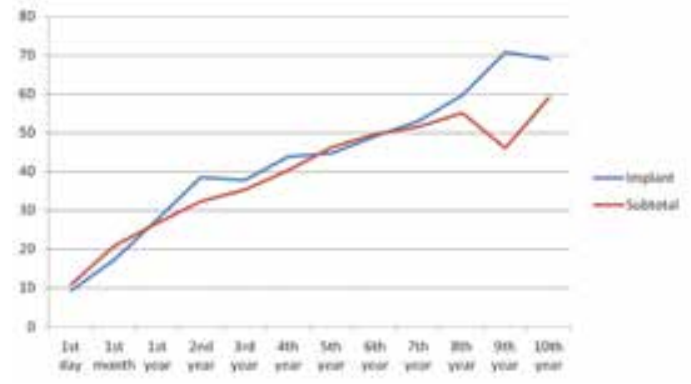

FIG. 1. Mean levels of intact parathyroid hormone according to study groups

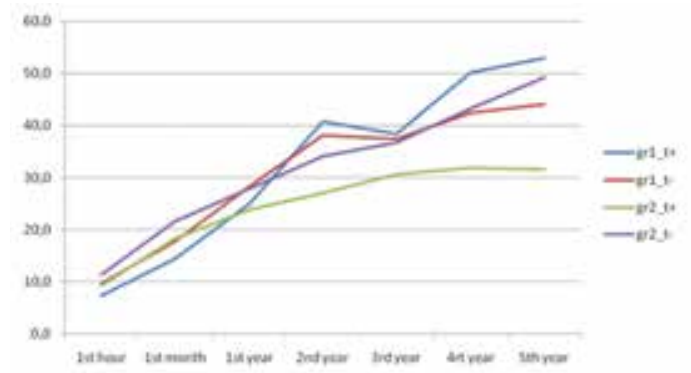

FIG. 2. Mean levels of intact parathyroid hormone in the $P T X+A(g r 1)$ and subtotal PTX (gr2) groups according to having thyroidectomy $(\mathrm{t}+)$ or no thyroidectomy $(\mathrm{t}-)$

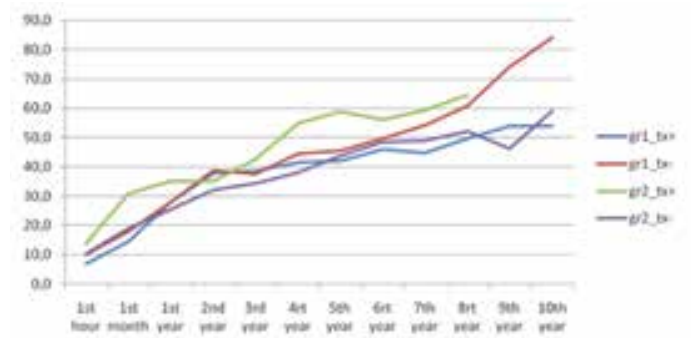

FIG. 3. Mean levels of intact parathyroid hormone in the groups according to renal transplantation status (gr 1: total PTX+AT, gr 2: subtotal $\mathrm{PTX}, \mathrm{tx}+$ : with transplantation, $\mathrm{tx}-$ : without transplantation)

result from many factors apart from the surgical technique. It is interesting that two patients with hypoparathyroidism from the subtotal PTX group later underwent total thyroidectomy. It may be speculated that perfusion of the remaining gland may be disturbed during thyroidectomy.

A dramatic drop in iPTH levels below normal has been reported after renal transplant and the recovery of normal renal function $(17,23)$. Successful transplantation was performed on $18 \%$ of our patients. This may also have affected the iPTH levels of both groups and the low rate of hyperparathyroidism. Intact PTH after total PTX may arise from hyperplasia of residual parathyroid fragments under continuing uremic stimula- tion; by renal transplantation this effect is compromised. The aim of this operation is to bring PTH levels under control, minimize the likelihood of recurrent disease and limit potential sites of recurrence. The rate of persistent or recurrent hyperparathyroidism is $0-26 \%$, and the rate of reoperation for recurrence is $5-15 \%$. Pattou et al. (24) reported that "supernumerary parathyroid glands are present in 30\% of patients with sHPT and are situated mainly in the thymus". This tissue is prone to hyperplasia when exposed to long-term stimulation. Thymectomy should be performed routinely during the first surgical exploration to prevent recurrences that might arise from the anterior mediastinal glands. It is worth noting that only Lorenz et al. (8) routinely performed cervical thymectomy in their 23 patients who underwent total parathyroidectomy without autotransplantation. The absence of PTH is associated with the risk of adynamic bone disease, especially in young patients who could receive a transplant. The presence of PTH following surgery suggests that some residual tissue remains and supports the practice of routine thymectomy (25). This may explain the low iPTH levels that we observed in follow-up. We believe that a complete inspection including the identification of parathyroid tissue combined with the excision of central fat while leaving a vascularized remnant in the neck or a transplant outside the neck is the procedure of choice for sHPT. Performing routine cervical thymectomy may decrease rates of persistence and the recurrence of PTH, as it did in our study group.

It is also well worth noting that all recurrences occurred in patients who were receiving dialysis treatment $(26,27)$. Therefore, the most important reason for recurrence was related not to the surgical approach itself but rather to the effects of longstanding renal disease, given that no recurrence was observed among patients who received renal transplantation, even throughout a long follow-up period. The rate of transplantation was $20 \%$ in our study group (16\% in the subtotal PTX group and $20 \%$ in the total PTX group). As the main etiologic factor for recurrence was eliminated in those patients, most of our patients' PTH levels have remained in a range at which no reoperations are required.

The strengths of our present study include its prospective randomized design, standardization of the surgical technique and long follow-up time. A limitation of our study is that it was inadequately powered to assess the differences between the groups because we included patients who underwent transplantation and whose etiologic susceptibility to recurrence was thereby abolished. Nevertheless, our study demonstrated favorable short-term and long-term outcomes throughout a follow-up period of long duration.

This study reviewed a group of secondary hyperparathyroidism patients prior to the use of calcimimetics. Although calcimimetics have probable and partially proven effectiveness in the clinical management of renal patients, they have only been available in Turkey for two years, and even now 
not all chronic renal disease patients can afford them. Even in kidney-transplanted patients, parathyroidectomy is an important therapeutic procedure, as it appear to safely preserve renal function regardless of the surgical technique. In this prospective randomized study, we found that patients who underwent total PTX+AT had lower rates of recurrence than those who had subtotal PTX; this is in agreement with current literature. We believe that, in order to avoid possible hypoparathyroidism and to manage persistent or recurrent HPT, total PTX+AT is the more suitable procedure for sHPT.

Ethics Committee Approval: Ethics committee approval was received for this study from the ethics committee of Çukurova University Faculty of Medicine, Adana, Turkey.

Informed Consent: Written informed consent was obtained from patients who participated in this study.

Peer-review: Externally peer-reviewed.

Author contributions: Concept - G.S., O.D.; Design - G.S., O.D., C.K.P.; Supervision - G.S.; Resource - G.S., O.D., C.K.P., I.C.E.; Materials - G.S., O.D., M.B.; Data Collection\&/or Processing - I.C.E., G.S., M.B.; Analysis\&/or Interpretation G.S.; Literature Search - G.S., O.D., C.K.P.; Writing - G.S.

Conflict of Interest: No conflict of interest was declared by the authors.

Financial Disclosure: The authors declared that this study has received no financial support.

\section{REFERENCES}

1. Malberti F, Marcelli D, Conte F, Limido A, Spotti D, Locatelli F. Parathyroidectomy in patients on renal replacement therapy: an epidemiologic study. J Am Soc Nephrol 2001;12:1242-8.

2. Tominaga Y, Uchida K, Haba T, Katayama A, Sato T, Hibi Y, et al. More than 1,000 cases of total parathyroidectomy with forearm autograft for renal hyperparathyroidism. Am J Kidney Dis 2001;38:168-71. [CrossRef]

3. Fassbinder W, Brunner FP, Brynger H, Ehrich JH, Geerlings W, Raine $\mathrm{AE}$, et al. Combined report on regular dialysis and transplantation in Europe, XX, 1989. Nephrol Dial Transplant 1991;Suppl 1:5-35.

4. Shih ML, Duh QY, Hsieh CB, Lin SH, Wu HS, Chu PL, et al. Total parathyroidectomy without autotransplantation for secondary hyperparathyroidism. World J Surg 2009;33:248-54. [CrossRef]

5. Hargrove GM, Pasieka JL, Hanley DA, Murphy MB. Short- and longterm outcome of total parathyroidectomy with immediate autografting versus subtotal parathyroidectomy in patients with end-stage renal disease. $\mathrm{Am}$ J Nephrol 1999;19:559-64. [CrossRef]

6. Gasparri G, Camandona M, Abbona GC, Papotti M, Jeantet A, Radice E, et al. Secondary and tertiary hyperparathyroidism: causes of recurrent disease after 446 parathyroidectomies. Ann Surg 2001;233:65-9. [CrossRef]

7. Naranda J, Ekart R, Pecovnik-Balon B. Total parathyroidectomy with forearm autotransplantation as the treatment of choice for secondary hyperparathyroidism. J Int Med Res 2011;39:978-87. [CrossRef]

8. Lorenz K, Ukkat J, Sekulla C, Gimm O, Brauckhoff M, Dralle H. Total parathyroidectomy without autotransplantation for renal hyperparathyroidism: experience with a qPTH-controlled protocol. World J Surg 2006;30:743-51. [CrossRef]

9. National Kidney Foundation K/DOQI clinical practice guidelines for bone metabolism and disease in chronic kidney disease. Am J Kidney Dis 2003;42:1-140. [CrossRef]
10. Santos RO, Ohe MN, Carvalho AB, Neves MC, Kunii I, LazarettiCastro M, et al. Total parathyroidectomy with presternal intramuscular autotransplantation in renal patients: A prospective study of 66 patients. J Osteoporos 2012;2012:631243.

11. Pitt SC, Sippel RS, Chen H. Secondary and tertiary HPT, state of the art surgical management. Surg Clin North Am 2009;89:1227-39. [CrossRef]

12. Riss P, Asari R, Scheuba C, Niederle B. Current trends in surgery for renal hyperparathyroidism (RHPT) an international survey. Langenbecks Arch Surg 2013;398:121-30. [CrossRef]

13. Mittendorf EA, Merlino JI, McHenry CR. Post-parathyroidectomy hypocalcemia: incidence, risk factors, and management. Am Surg 2004;70:114-19.

14. Drueke TB, Ritz E. Treatment of secondary hyperparathyroidism in CKD patients with cinacalcet and/or vitamin D derivatives [Review]. Clin J Am Soc Nephrol 2009;4:234-41.[CrossRef]

15. Wallfelt CH, Larsson R, Gylfe E, Ljunghall S, Rastad J, Akerström G. Secretory disturbance in hyperplastic parathyroid nodules of uremic hyperparathyroidism: implication for parathyroid autotransplantation. World J Surg 1988;12:431-8.[CrossRef]

16. Riss P, Kaczirek K, Heinz G, Bieglmayer C, Niederle B. A "defined baseline" in PTH monitoring increases surgical success in patients with multiple gland disease. Surgery 2007;142:398-404. [CrossRef]

17. Bieglmayer C, Kaczirek K, Prager G, Niederle B. Parathyroid hormone monitoring during total parathyroidectomy for renal hyperparathyroidism: pilot study of the impact of renal function and assay specificity. Clin Chem 2006;52:1112-9. [CrossRef]

18. Pitt SC, Panneerselvan R, Chen H, Sippel RS. Secondary and tertiary hyperparathyroidism: the utility of ioPTH monitoring. World $J$ Surgery 2010;34:1343-9. [CrossRef]

19. Riss P, Asari R, Scheuba C, Niederle B. Current trends in surgery for renal hyperparathyroidism (RHPT) -an international survey. Langenbecks Arch Surg 2013;398:121-30. [CrossRef]

20. Agha A, Loss M, Schlitt HJ, Scherer MN. Recurrence of secondary hyperparathyroidism in patients after total parathyroidectomy with autotransplantation: technical and therapeutic aspects. Eur Arch Otorhinolaryngol 2012;269:1519-25. [CrossRef]

21. Kinnaert P, Salmon I, Decoster-Gervy C, Vienne A, De Pauw L, Hooghe $\mathrm{L}$, et al. Long-term results of subcutaneous parathyroid grafts in uremic patients. Arch Surg 2000;135:186-90. [CrossRef]

22. Stracke S, Keller F, Steinbach G, Henne-Bruns D, Wuerl P. Long-term outcome after total parathyroidectomy for the management of secondary hyperparathyroidism. Nephron Clin Pract 2009;111:c102-9. [CrossRef]

23. Triponez F, Dosseh D, Hazzan M, Noel C, Soudan B, Lokey J, et al. Accuracy of intra-operative PTH measurement during subtotal parathyroidectomy for tertiary hyperparathyroidism after renal transplantation. Langenbecks Arch Surg 2006;391:561-5. [CrossRef]

24. Pattou FN, Pellissier LC, Noël C, Wambergue F, Huglo DG, Proye CA. Supernumerary parathyroid glands: frequency and surgical significance in treatment of renal hyperparathyroidism. World J Surg 2000;24:1330-4. [CrossRef]

25. Schneider R, Waldmann J, Ramaswamy A, Fernández ED, Bartsch DK, Schlosser K. Frequency of ectopic and supernumerary intrathymic parathyroid glands in patients with renal hyperparathyroidism: analysis of 461 patients undergoing initial parathyroidectomy with bilateral cervical thymectomy. World J Surg 2011;35:1260-5. [CrossRef]

26. Rayes N, Seehofer D, Schindler R, Reinke P, Kahl A, Ulrich F, et al. Long-term results of subtotal vs total parathyroidectomy without autotransplantation in kidney transplant recipients. Arch Surg 2008;143:756-61. [CrossRef]

27. Richards ML, Wormuth J, Bingener J, Sirinek K. Parathyroidectomy in secondary hyperparathyroidism: Is there an optimal operative management? Surgery 2006;139:174-80. [CrossRef] 